\title{
Spectrum of Blast Injury
}

\author{
Bajracharya U.'
}

\section{Introduction}

The new millennium has commenced with a reminder that terrorist attacks and subsequent mass causality situation can occur anywhere and to any one. No one is immune. Terrorism information usually emphasizes on nuclear, chemical, biological, and radiation events. However, most terrorist attacks have involved explosive devices resulting in different spectra of blast injury. Such events are common these days in countries like Israel and Iraq, Nepal being no exception. Mortality has been found to be more after explosion than after other mentioned events. September 11 th 2001 event, 1995 Oklahoma bombing \& March 11th 2004 Madrid train explosion are few examples of mass causality situation related blast injuries. Internationally incidence is sporadic. Frequency depends on the political stability of the region. Mortality rates vary widely. Blast injuries were once rare during peace time, but the wide spread of terrorism has made no nation immune to it.

\section{Types of Explosive:}

1) Conventional Bombs

2) Enhanced Blast Explosive Device

1. Conventional Bombs: - This explosion generates a blast wave that spreads out from a point source. This wave consists of two partsa shock wave of high pressure, followed closely by a blast wind. The physics of this wave is non-linear and complex. Normally damage by this wave decreases exponentially with distance from the point source of the blast. During indoor explosion, standing waves and enhanced pressure differences occur due to the additive reflections from walls and rigid objects. As energy dissipates outwards, a reversal of wind back towards the blast occur resulting under-pressurization.

2. Enhanced Blast Explosive Device:- This explosion has more damaging effects. A

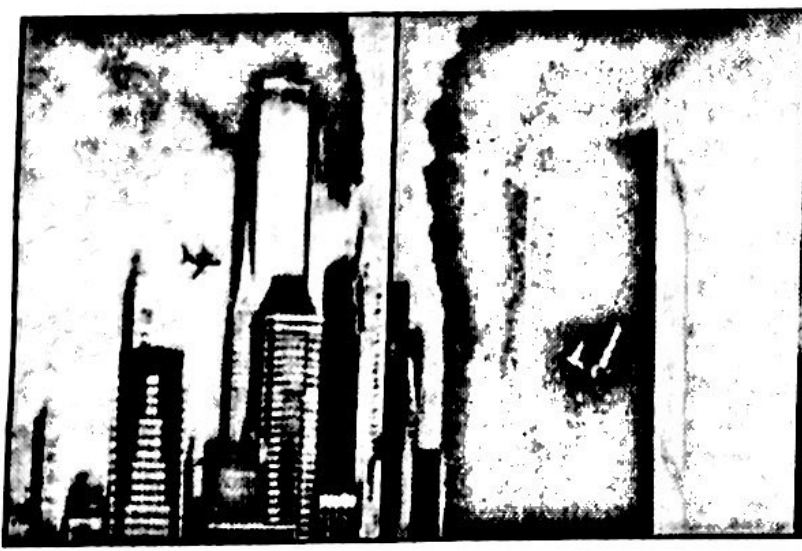

Figure 1 Showing 9/11 Episode

\section{Classification of Explosives}

Explosives can be classified in different ways. Few are mentioned below:-

1. Dr. Uday Bajracharya, M.B.B.S, MD, Maj., Consultant Anesthesiologist, SBH Assistant Professor, NAMS

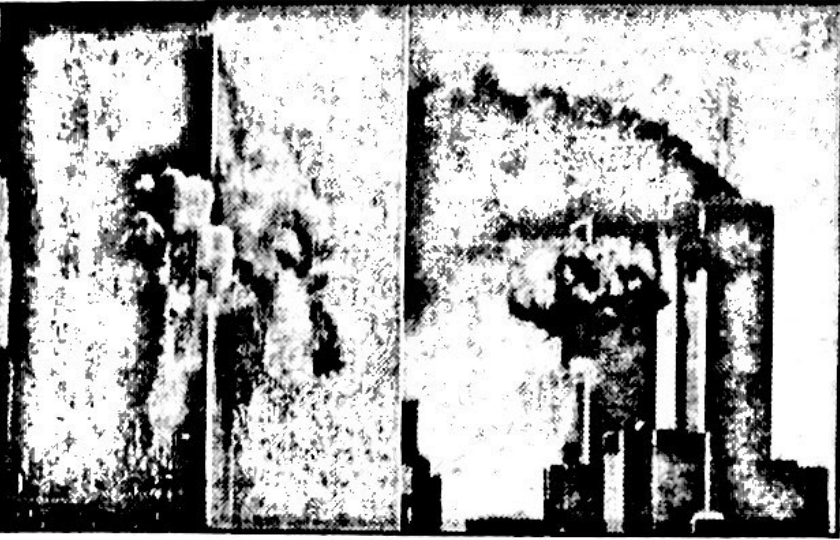

primary blast disseminates the explosive and then triggers it to cause a secondary explosion. The high-pressure wave then radiates from a much larger area, prolonging the duration of the over pressurization phase, increasing the total energy transmitted by the explosion.

Explosive can also be categorized according to the rate of the decomposition (in sec, $\mathrm{min}$, hrs, days, month, yrs). 
1) Low order Explosive

2) High order Explosive

1) Low order Explosive (LOE): - It burns rapidly (Deflagrate). Blast wave has subsonic speed (upto $400 \mathrm{~m} / \mathrm{sec}$ ).Decomposition is propagated by a flame front, which slowly travels through the explosive materials. e.g. propellants, mixtures(gun powder) smokeless powder, pyrotechnics, pyrogenic flares \& illumination devices, pipe bombs, Pure Petroleum based bombs.(Molotov Cocktails, aircraft improvised as guided missiles).

2) High order Explosive (HOE):- It undergoes detonation. The Blast wave has supersonic speed (upto $9-20 \mathrm{~km} / \mathrm{sec}$ ). Decomposition is propagated by explosive shock wave penetrating explosive materials. e.g. TNT, C4, Semtex, Nitroglycerin, Dynamite and Ammonium Nitrate Fuel Oil (ANFO).

HOE \& LOE involve ballistic and thermal injuries. $\mathrm{HOE}$ also involves blast wave injuries which are characterized by anatomical and physiological changes from over-pressurization force (OPF) on body surface. The HOE-blast wave (OPF) is different from blast-wind (forced super-heated airflow) that may be encountered with both HOE and LOE.

Ideally, blast is detonation of HOE in bombs, shells, grenades or mines, \& has concussive effects. Blast wave has about $3 \mathrm{~km} / \mathrm{sec}$ of speed in all directions. The pressure of blast-wave is about $20 \mathrm{~kg} \mathrm{~cm}-2$ $(=40,000 \mathrm{~kg}$ on trunk of a man). Air absorbs blastwave well and rapidly react with increasing range from detonated point. HOE exhibits rapid explosion (rapid production of gases with rapid heating surroundings), evolution of heat, initiation of reaction and rapidity of reaction. Under- water blast consist of initial shock wave imparted to water. Pulse produced by successive collapse of bubble causes turbulence \& thursting of surrounding water.

\section{CLASSIFICATION OF BLAST INJURY}

There are Five Basic Mechanisms of Blast Injury
a. Primary
b. Secondary
c. Tertiary
d. Quaternary
e. Quinary

Primary Blast Injury (PBI) has characteristics unique to detonated HOE, resulting from the intense impact of the over pressurization wave (HOE blast wave) with body surfaces. It affects gas air interfaces. Organs damaged by dynamic pressure changes at tissue density (air fluid) interfaces due interaction of high \& low frequency stress waves. Therefore, body parts affected are gas filled structures e.g. Lungs, GI Tract, and middle ear. Injuries include blast lung (pulmonary barotraumas), tympanic membrane (TM) rupture and middle ear damage, abdominal hemorrhage and perforation, globe (eye) rupture \& concussion (TBI without signs of head injury), arterial gas embolism(AGE), free radical injuries like thrombosis, lipo-xygenation, disseminated intravascular coagulation (DIC) \& adult respiratory distress syndrome(ARDS)

Secondary Blast Injury refers to characteristics that result from flying debris and bomb fragments. Any part of the body may be injuries include penetrating ballistic (Fragmentation) or blunt injuries \& Eye penetration (can be occult).

Tertiary Blast Injury has a feature of high-energy explosion \& results from individuals being thrown by the blast wind which is the forced super- heated airflow.

Any part of the body may be injuries include fracture and traumatic amputation and closed and open brain injury, crush injury and compartment syndrome. It also includes deceleration injury

Quaternary Blast Injury refers to all explosion related injuries, illnesses, or diseases not due to primary, secondary or tertiary mechanisms. It includes exacerbation or complications of existing conditions (e.g pregnancy\& anti coagulation). Body part affected may be any part of the body. Injuries include burns (Flash, Partial, and full thickness), (thermal and chemical) crush injuries, closed and open brain injury, asthma, COPD, or other breathing problems from dust, smoke, or toxic fumes, angina, hyperglycemia, hypertension.

Quinary Blast Injury results from absorbed micro substance e.g. chemical contamination and irradiation of explosion victims. Body Part affected may be any part of the body. Injuries include skin problems, hematological problems, neurogical problems etc.

\section{Nature of the Blast Pressure Wave}

Explosions produce unique patterns of injury rarely seen outside combat. When they occur, they have the potential to cause multi- system life threatening 
injuries on many persons simultaneously. The injury patterns following such events are a product of the composition and amount of the materials involved, the surrounding environment, delivery method (if a bomb), the distance between the victim and the blast.

\section{Blast Physics}

An underwater blast is more dangerous than an air blast for an explosion with the same energy and at the same distance because the blast dissipates in air and tends to be reflected at body surface, where as in water the blast wave travel through the body and causes internal gas liquid interface organ damage.
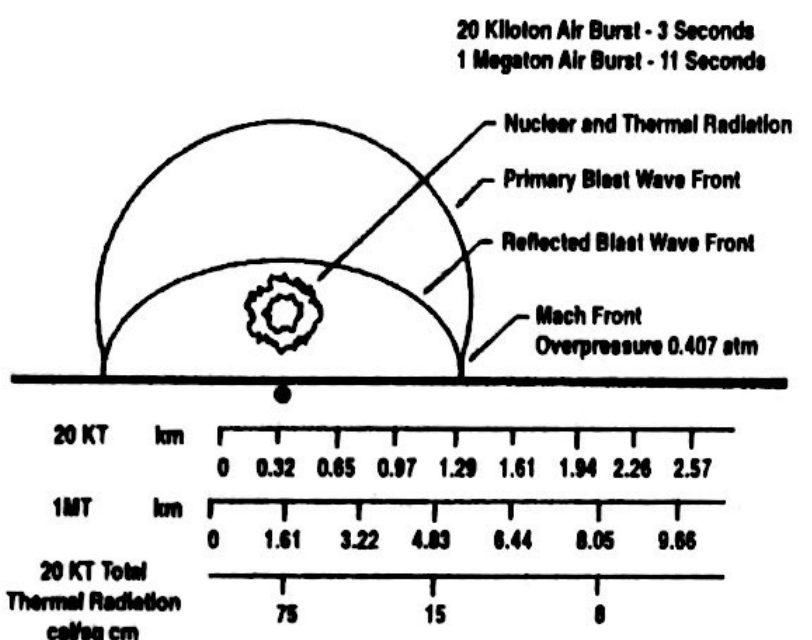

Figure 2 showing chronological development of air blast
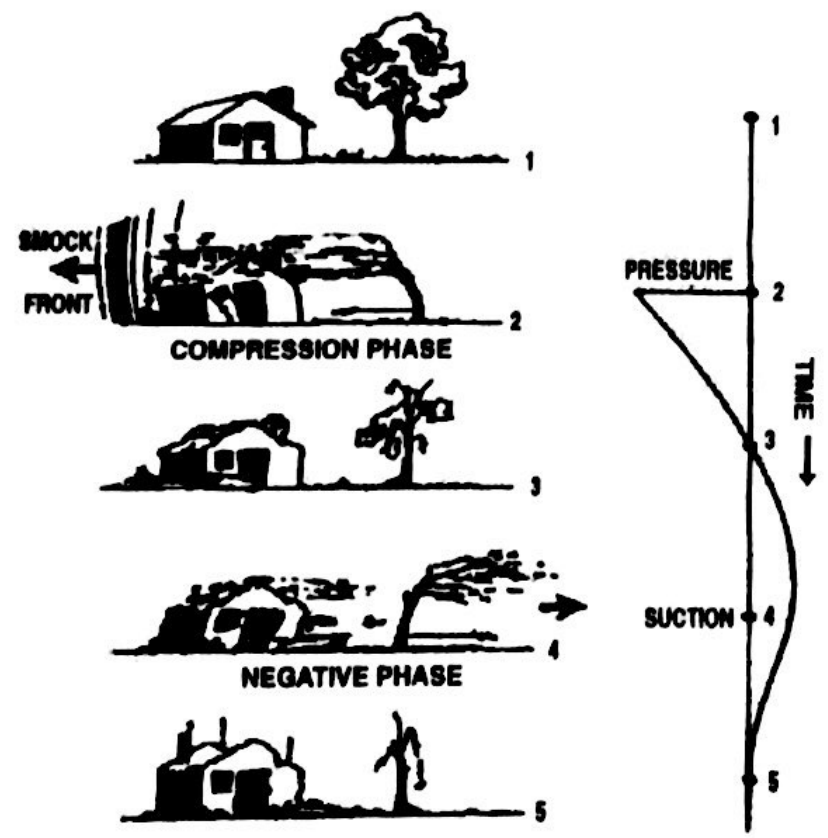

Figure 3 showing variation of blast effects associated with positive \& negative phase pressure with time
The damaging effect of the explosion depends on the peak of the pressure wave \& the duration of the pressure wave. These variables in turn depend upon nature of charge size of charge, and distance from charge.

The initial pressure change of the blast wave is steep, rising to a peak pressure within milliseconds. The pressure in the bubble falls as it expands \& the gas cools. The fall of pressure at the end of the explosion reflects the end of the expansion of the gas and take milliseconds. The pressure is then less than previous ambient pressure. Most organ damage is due to the primary shock wave, burning $\&$ does not have brisance

\section{Hollow Viscous Blast Injuries (PBI to GI Tract)}

Manifestations typically overshadowed more by life threatening pulmonary PBI. There is evidence at GI injury can occur at same time or before pulmonary PBI. Gas containing organs are more commonly affected than solid intra abdominal organs. Damage to solid organs more likely from secondary and tertiary blast injury. Lesions include edema, hemorrhage and organ rupture. Patients present with abdominal pain nausea and vomiting, diarrhea, tenesmus. Signs may be absent bowel sounds, guarding and rebound tenderness, haematemesis and haematochezia. There may be rupture of small bowel (few), large bowel, and rectum (Haemoperitoneum). There are very little reports about solid organ injuries. Many cases have serosal ecchymoses only. Immersion blast is worse than air. Under- water blast causes damage to organs eg. Spleen, liver. Effects of PBI to GI Tract has been reported as late as two weeks.

\section{Auditory Apparatus Blast Injury (Auditory PBI)}

The most vulnerable organ is auditory apparatus. Damage occurs to both middle and inner ear. leading to hearing loss(+). Less common manifestations include otalgia, tinnitus or vestibular dysfunction leading to vertigo Injuries varies from slight reddening to complete perforation of ear drum.\& formation and development of perilymphatic fistulae. The cochlea can be damaged even if ear - drum stays intact, ossicular disruption, FB ear. Perforation may occur with pressure as low as 5 p.s.i \& is variable between victims. 


\section{Primary Blast Lung( PulmonaryPBI)}

Blast effect damage is not by traveling along the trachea and bronchi. It passes through the body wall. (15psi). Shredding effects at the tissue -air interface causes intra - alveolar haemorrhage with resultant ventilation -perfusion mismatch. Neutrophilic inflammatory reaction occurs (if patients survives) leading to pneumonitis, sepsis, hemorrhage and eosinophilic edema in alveolar spaces. Degree of respiratory insufficiency depends on degree of hemorrhage. Laceration of hilar tissue (vessels, bronchi) may occur leading to haemoptysis. Hemothorax(HTX) can occur with parenchymal laceration. Lung is traditionally considered the non -auditory organ most at risk for suffering PBI. Damage includes pulmonary contusion, with or without laceration, and / or pulmonary barotraumas including broncho-pulmonary fistula. Contusions may develop/stabilize/resolve rapidly. Barotrauma results from local changes within the thorax leading to tearing of the alveolar septae.( Pulmonary Infarction, Embolism, Pneumomediastinum, Subcutaneous emphysena, Pneumothorax (PTX), AGE. Patients may present with dyspnea, chest pain, hemoptysis, cough. On examination, Signs are tachypnea, cyanosis, decreased breath sounds and dullness to percussion, increased resonance, subcutaneous crepitus, cardiovascular collapse. Pulmonary PBI is triad of bradycardia, hypotension and apnoen, mediated by vagal reflexes. Blast lung produces a characteristic "butterfly" pattern on chest $X$-ray. Pulmonary injuries with the immediate on- set of pulmonary edema frothing at the mouth (associated with bilateral radiographic" whiteout") carries a grave prognosis. Effects of PBI has been reported as late as one week.

\section{Short Duration Pressure Effects Upon Unprotected Persons.}

$\begin{array}{ll}\text { Pressure (PSI) } & \text { Effect } \\ 5 & \text { ? Ear-drum rupture } \\ 5 & 50 \% \text { Ear-drum rupture } \\ 33-40 & \text { Slight chance of lung injury } \\ 80 & 50 \% \text { of chance of lung injury } \\ 100-200 & \text { Slight chance of death } \\ 130-180 & 50 \% \text { death } \\ 200-250 & \text { Death Usually } \\ 15 \mathrm{PSI}=1 \mathrm{~kg} / \mathrm{cm} 2=100 \mathrm{Kpa}=760 \\ \mathrm{mmHg}=1000 \mathrm{cmH} 20=1 \mathrm{bar} / 1 \mathrm{ATM}\end{array}$

Primary Blast Injury after bomb explosion in a civilian bus. Ann Surg 1989

The researchers of the study of 647 survivors of explosions on buses used immediate radiography of the chest to screen for pulmonary injuries from the blasts. Primary injuries, in some form, were found in 193 persons: 142 has isolated perforation of the eardrum, and 51 had other form of primary blast injuries, including 18 with isolated pulmonary injuries, 31 with combined otic and pulmonary injuries, and 2 with intestinal injuries.

\section{Madrid Bombing 1995: (243 victims)}

In recent report of the train bombing in Madrid, rupture of the tympanic membranes occurred in 99 of 243 victims, chest injuries in 97 , shrapnel wounds in 89 , fractures in 44 , burns in 45 , eye injuries in 41 , abdominal injuries in 12 , and traumatic amputation in 5 . Among 17 critically ill victims with pulmonary injuries from the blast, 13 had ruptured tympanic membranes. Rupture of tympanic membranes occurred in 18 of 27 critically injured victims; 17 of these were bilateral.

\section{Two Bus bombings in Israel 1996:}

In two bus bombings in Israel described in 1996, 22 of 52 injured survivors (42 percent) needed endotracheal intubation and $10(19$ percent) required chest tube thorcostomies. After two open air bombings in Israel that year, only 1.3 of 190 victims ( 7 percent) were intubated, and $5(3$ percent) required chest tubes

Ref: Blast injuries; Bus versus open air bombings Journal of Trauma 1996, 41

\section{Management}

The treatment of blast injuries, whether combined with other injurers or not, is best managed by applying accepted principles of combat surgery. Treatment is divided into four basic plans.

a) Resuscitative Phase: Lifesaving resuscitative measures are prepared for definitive surgical treatment. These include the establishment of the airway assuring the adequacy of respiration, replacement of lost blood and fluids, and splinting of possible fractures, particularly those involving the cervical vertebrae. Resuscitative measures should start prior to evacuation from the battlefield. 
b) Surgical Phase: Definitive surgery should be done after resuscitative measures have been used to improve the patient's condition to minimize the risk of surgery and anesthesia. Occasionally, lifesaving surgery must be done without delay, but normally there is time to prepare patients for surgery if they have survived long enough to reach a treatment facility.

c) Recovery phase: In the immediate postoperative period, patients require minimal movement. Transportation to other facilities should be delayed until the patient's condition has stabilized.

d) Convalescent Phase: Patient in this phase of treatment should be evacuated back to specialized convalescent facilities to keep the patient load as low as possible. Many injuries may require a prolonged recovery period before the individual has recovered to the point where he /she can resume their duties. Both the convalescent and recovery phases will be more protracted with the addition of the radiation injury.

History should include about distance from explosion, exposure under water or in an enclosure $\&$ body armor worn by victim

\section{Investigation \& Treatment:}

\section{Investigations}

a. Hb, PCV for bleeding, Otoscopy and Audiometry in case of TM rupture.

b. Urine Analysis in case of Myoglobinuria (crush injury)

c. Carboxy Hemoglobin in $\mathrm{CO}$ inhalation(SPO2 reading may be misleading)

d. CN Hemoglobin \& ABG (anion gap metabolic acidosis) in case of cyanide-poisoning

e. Coagulation profile in case of DIC, $\mathrm{K}+\&$ PHO24 level in case of white phosphorus burns.

f. CXR PA view in case of Blast Lung

g. X-ray Abdomen (Pneumo-hemoperitoneuim)

h. CT/MRIAbdomen/ Head/Thorax. (If History \& Examination suggest pathology or victim unconscious)

\section{Treatment:}

\section{Pulmonary PBI}

Initiation of life support (Primary and secondary survey) should be performed. Correction of the effects of barotraumas should be done to support gas exchange.

Limitation of physical activities must be advised. Air evacuation may worsen barotraumas. Marginal Oxygenation will worsen with altitude. Tube thoracostomy(chest tubes)is advised for tension PTX, Aircraft cabin is to be pressurized when flying at 5000 to 8000 feet. Air transport by low altitude helicopter is necessary to prevent expansion of air emboli.

Risks of mechanical ventilatory support (PPV \& PEEP) are reduced cardiac output, arterial air embolism and thoracic barotraumas. Therefore, supplemental oxygen and non- invasive ventilation have to be instituted. If ET intubation is necessary, ventilatory parameters are set to maximize mean airway pressure and minimize peak airway pressure (ARDS patients). Hypotension may be because of blood loss secondary to injury, GI hemorrhage or solid organ rupture, sequelae of air embolism, \& vegal reflexes.

Resuscitation is considered by instituting appropriate fluids ensuring tissue-perfusion without volume overload and central monitoring .Arterial air embolism is responsible for most early sudden deaths. It results from alveolo-venous fistulae leading to obstruction in coronary/cerebral circulations. These are only casualties with clinical evidence of pulmonary contusion who are at risk. Arterial air embolism inay present with blindness or focal neurological deficit, chest pain and loss of consciousness. Signs are air in retinal vessels (ophthalmoscopy), focal neurologic deficits and tongue blanching or livedo reticularis. Arterial air embolism can be managed by supplemental high oxygen flow (non-rebreathing masks, CPAP mask, and ETT), appropriate body position, mechanical ventilation, and hyperbaric oxygen therapy. Body can be placed in left lateral decubitus position with head down. Injured lung should be placed in dependent position if unilateral lung injury occurs.

\section{GI PBI:}

Initial approach is same as that for pulmonary PBI. If victim is conscious and there is no sign of peritoneal irritation, follow up is enough. If victim 
is hemodynamically stable, and conscious with abdominal findings, or unconscious, then abdominal CT with oral and IV contrast can be done. Following findings on CT suggest for exploratory laparatomy.(Ex-lap)

I. Extraluminal gas or contrast.

2. Hemoperitoneum

3. Solid organ injury/ disruption

4. Hypodense accumulation of fluid, including bowel contents

5. Bowel wall hematoma

CT is $90 \%$ specific \& $25-75 \%$ sensitive (hollow viscous injury frequently missed)

If patient still presents with sign of peritoneal injury after negative CT, diagnostic peritoneal lavage (DPL) can be performed.

a. If $>10 \mathrm{ml}$ of non-clothing blood is aspirated, proceed for ex- lap

b. If aspirate is negative, 1 liter of saline is infused into abdomen and allowed to drain out by gravity.

c. If $>105 \mathrm{RBC} / \mathrm{ml}, 500 \mathrm{WBC} / \mathrm{ml}$, bacteria, bile or fecal matter, return to ex-lap.

d. DPL is far more sensitive and has equivalent specificity to abdominal CT.

If patient has abdominal complaints and negative abdominal CT and DPL, then monitor closely as episodes of peritonitis or intra abdominal abscess formation may occur within days to weeks. If victim is hemodynamically unstable and conscious with abdominal findings, or unconscious, DPL can be carried out while resuscitating .If DPL is positive, proceed for ex- lap. If DPL is negative/ hypotension resolves, follow up is required. If DPL is negative and hypotension is persists, ex lap can be performed as DPL can miss retroperitoneal injury, mesenteric hematoma or solid organ injuiries. If abdominal CT is to be done, it must precede any attempt at DPL because of false positive CT. If Ex - lap is planned, one must obtain CXR prior to surgery, looking for evidence of pulmonary barotruama.

\section{Auditory PBI}

There is no specific therapy shown to aid recovery from acoustic trauma. Treatment must begin with avoidance of further auditory injury. For TM rupture, remove debris from the external canal and irrigate canal with an antiseptic solution. Primary Closure is performed if $>1 / 3 \mathrm{TM}$ is involved. Therefore, one should be aware of the possibility of PBI among those who have secondary and tertiary blast injuries. Damage to the air containing organs of the thorax and abdomen cause the most life threatening PBI. Injury to non- gas containing organs is critical and requires more investigation.

Decontamination of nerve gases should be done in case of chemical warfare. White phosphorus contaminated burns needs copious lavage of the area using Wood lamp in dark room and rinse using $1 \%$ Copper sulfate solution, keeping in mind that excess CuSO 4 absobption can cause renal failure.In case of $\mathrm{CN}$ (Cyanide poisoning), thiosulfate $(25 \%)$ Hydroxy -Cobalamin (BI2) or Sodium Nitrite (3\%) can be used.

FFP\& Platelets can be used in case of DIC.

\section{Special Concern:}

Pregnant patients with blast injuries warrant special consideration. Direct injury to the fetus is uncommon as the fetus floats on the incompressible amniotic fluid. The placental attachment is at risk for primary blast injury because of the effects passing from a higher density medium (endometrial muscle) to a lower density damaging tissues at the interface. In addition, tissues of different densities are affected causing shearing injuries and placental abruption. Kleihauer - Betke assay (screening test for feto maternal hemorrhage) should be obtained. A positive test (detection of fetal cells in the maternal bloodstream) suggest for obstetric consultation.

\section{Conclusion:}

Bombs and explosions cause unique pattern of injury hardly seen outside combat. The predominant post-explosion injuries are standard peneratrating and blunt trauma. Blast lung is the most common fatal injury among survivors. Explosions in confined spaces (mines, buildings, or large vechicles) involve greater morbidity and mortality. Half of all initial casualties seek medical care over a one hour period. This can be predict demand for care and resource needs. One must expect an "upside -down" triage - the most severely injured arrive 
after the less injured.Most deaths are immediate. ERdeaths are statistically $1 \%$ or less.Usually the bottleneck in the ER will be the radiological screening of noncritical victims.

\section{REFERENCES:}

1. Blast injuries bus versus open air bombings a comparative study of injuries in survivors of open-air versus confirmed space explosions $\mathbf{J}$ Trauma 41(6): 1030-5,1996.

2. http:/www.themelalith.com/blastinjuries.html

3. http:/www.nbc-med.org/Site Content/ Med Ref/ Online Ref/ Field Manuals/amedp6/P.../ chapter4 html.

4. http:/www.emedicine.com/EMERG/ topic63.html.

5. Blast injuries: Ralph G. DePalma et at, The New England Journal of Medicine Volume: 352 Number 13, 2005

6. Wightman JM, Gladish SL. Explosion and blast injuries:

A primer for clinicians. Atlanta: Centres for Disease Control and prevention (March 7,2005).

7. Treatment of Primary Blast injury in emergency war surgery NATO hand book (March 10,2005).

8. Horrocks CL Blast injuries; Biophysics, pathophysiology, and management principles J.R. Corps 2001: 147 : 28-40

9. Langworthy MJ, Sabra J. Gould M. Terrorism and Blast Phenomena: lessons learned from on the USS Cole (DDG67). Clinic Orthop. 2004:422: 82-87

10. Elsayed NM. Texicology of Blast Overpressure. Toxicology. 1997;121: 121-15

11. Mayor MA. The pathology of primary blast overpressure injury. Toxicology. 1997; 121

12. Sasser SM, Blast injuries. TheTukish Journal of Emergency medicine.2001;(1):97-98

13. Philips YY. Primary blast injuries. Ann Emerg Med.1986; 15:1446-1450

14. Katz E, Ofek B, Alder J, et a. Primary blast injury after a bomb explosion on a civilian bus 1989;209(4):484-488.
15. De ceballos JPG, Turegano-Fuentes F, PerezDiaz D at et al: II March 2004. The terrorist explosions in Madrid, Spain an analysis of the logistics, injuries sustained and clinical manag casualties treated at the closest hospital. Crit care Med. 2005 Jan; 33(1):s 107-112.

16. Leibovici D, Gofrit ON, stein M, et al .Bast injuries; bus versus open - air bombings a com study of injuries in survivors of open-air versus confined space explosions. J. Trauma 1996; 1035.

17. Irwin RJ, Lerner MR, Bealer JF, Mantor PC et al. Shock after blast wave injury is caused vegally mediated reflex. J.Trauma 1999.;47(1) 105-110

18. Argyros, GJ, Management of Primary Blast Injury. Toxicology, 1997 121;105-115

19. Irwin RJ, Lerner MR, Bealer JF, etal . Cardiopulmonary physiology of primary blast injury. 1997; 43(4);650-655.

20. Pattern of global terrorism 2001, 2002, 2003 United States of state May 2002, April 2003, April 2004 United State Department of State website. Available at: http:// www.state.gov/ documents/organization/103,201, 319 Accessed 4 February 2005.

21. Federal Bureau of investigation. 1999 Bombing incidents. Washington, D.C Department of Justice, 2003 (FBI Bomb Data Center publication no.0367).

22. Cullis IG. Blast waves and how they interact with structures. JR Army Med corps 2001; 147; 16-26.

23. Guy RJ, Glover MA, Cripps NP. The path physiology of primary blast injury and its implications for treatment. Part I; the thorax. JR Nav Med serv 1998;79-86

24. Katz. E. Ofek B, Adler J, Abromowitz HB Krausz MM. Primary blast injury after a bomb explosion in a civilian bus. Ann Emerg, Med 1999; 34:168-172.

25. Irwin RJ, Lerner MR, Bealer JF, Mantor PC, Brackett DJ, Tuggle DW. Shock after blast wave injury is caused by a vagally mediated reflex. J. Trauma 1999; 105-11 